Collect. Math. 61, 3 (2010), 253-262

(c) 2010 Universitat de Barcelona

DOI $10.1344 / \mathrm{cmv} 61 \mathrm{i} 3.5384$

\title{
Weighted estimates for the averaging integral operator
}

\author{
Bohumír Opic ${ }^{1}$ \\ Institute of Mathematics, AS CR, Žitná 25, 11567 Praha 1, Czech Republic \\ E-mail: opic@math.cas.cz \\ JiŘí RÁKOSNÍK \\ Institute of Mathematics, AS CR, Žitná 25, 11567 Praha 1, Czech Republic \\ E-mail: rakosnik@math.cas.cz
}

Received July 29, 2009. Revised November 5, 2009

\begin{abstract}
Let $1<p \leq q<+\infty$ and let $v, w$ be weights on $(0,+\infty)$ satisfying:

$(\star) \quad v(x) x^{\rho}$ is equivalent to a non-decreasing function on $(0,+\infty)$ for some $\rho \geq 0$

and

$$
[w(x) x]^{1 / q} \approx[v(x) x]^{1 / p} \text { for all } x \in(0,+\infty) .
$$

We prove that if the averaging operator $(A f)(x):=\frac{1}{x} \int_{0}^{x} f(t) \mathrm{d} t, x \in(0,+\infty)$, is bounded from the weighted Lebesgue space $L^{p}((0,+\infty) ; v)$ into the weighted Lebesgue space $L^{q}((0,+\infty) ; w)$, then there exists $\varepsilon_{0} \in(0, p-1)$ such that the operator $A$ is also bounded from the space $L^{p-\varepsilon}\left((0,+\infty) ; v(x)^{1+\delta} x^{\gamma}\right)$ into the space $L^{q-\varepsilon q / p}\left((0,+\infty) ; w(x)^{1+\delta} x^{\delta(1-q / p)} x^{\gamma q / p}\right)$ for all $\varepsilon, \delta, \gamma \in\left[0, \varepsilon_{0}\right)$.
\end{abstract}

Conversely, assuming that the operator

$$
A: L^{p-\varepsilon}\left((0,+\infty) ; v(x)^{1+\delta} x^{\gamma}\right) \rightarrow L^{q-\varepsilon q / p}\left((0,+\infty) ; w(x)^{1+\delta} x^{\delta(1-q / p)} x^{\gamma q / p}\right)
$$

\footnotetext{
${ }^{1}$ The paper was supported by the grants nos. 201/05/2033 and 201/08/0383 of the Czech Science Foundation and by the Institutional Research Plan no. AV0 Z10190503 of the Academy of Sciences of the Czech Republic

Keywords: Averaging integral operator, weighted Lebesgue spaces, weights

MSC2000: Primary 26D10; Secondary 46E30
} 
is bounded for some $\varepsilon \in[0, p-1), \delta \geq 0$ and $\gamma \geq 0$, we prove that the operator $A$ is also bounded from the space $L^{p}((0,+\infty) ; v)$ into the space $L^{q}((0,+\infty) ; w)$. In particular, our results imply that the class of weights $v$ for which $(\star)$ holds and the operator $A$ is bounded on the space $L^{p}((0,+\infty) ; v)$ possesses properties similar to those of the $A_{p}$-class of $\mathrm{B}$. Muckenhoupt.

\section{Introduction}

Let $1<p<+\infty$ and let $v$ be a weight on $(0,+\infty)$, i.e., a measurable function which is positive a.e. on $(0,+\infty)$. By $L^{p}(v) \equiv L^{p}((0,+\infty) ; v)$ we denote the weighted Lebesgue space of all measurable functions $f$ on $(0,+\infty)$ for which the norm

$$
\|f\|_{p, v}=\left(\int_{0}^{+\infty}|f(x)|^{p} v(x) \mathrm{d} x\right)^{1 / p}
$$

is finite.

We shall consider one of very important operators in the mathematical analysis, the averaging operator $A$ defined by

$$
(A f)(x):=\frac{1}{x} \int_{0}^{x} f(t) \mathrm{d} t, \quad x \in(0,+\infty) .
$$

It is well known (see $[2,9])$ that if $1<p<+\infty$ and $w, v$ are weights on $(0,+\infty)$, then the averaging operator $A: L^{p}(v) \rightarrow L^{q}(w)$ is bounded if and only if

$$
B:=\sup _{r>0}\left(\int_{r}^{+\infty} w(t) t^{-q} \mathrm{~d} t\right)^{1 / q}\left(\int_{0}^{r} v(t)^{1-p^{\prime}} \mathrm{d} t\right)^{1 / p^{\prime}}<+\infty
$$

where $p^{\prime}=p /(p-1)$.

Throughout the paper we use the following convention: For two non-negative expressions (i.e. functions or functionals) $F$ and $G$ the symbol $F \lesssim G$ (or $F \gtrsim G$ ) means that $F \leq c G$ (or $c F \geq G$ ), where $c$ is a positive constant independent of appropriate quantities involved in $F$ and $G$. We shall write $F \approx G$ (and say that $F$ and $G$ are equivalent) if both relations $F \lesssim G$ and $F \gtrsim G$ hold.

Our main results are the following two theorems.

\section{Theorem 1.1}

Let $1<p \leq q<+\infty$ and let $v, w$ be weights on $(0,+\infty)$ such that:

$v(x) x^{\rho}$ is equivalent to a non-decreasing function on $(0,+\infty)$ for some $\rho \geq 0$;

$$
[w(x) x]^{1 / q} \approx[v(x) x]^{1 / p} \text { for all } x \in(0,+\infty) .
$$

Assume that the averaging operator $A: L^{p}(v) \rightarrow L^{q}(w)$ is bounded. Then there exists $\varepsilon_{0} \in(0, p-1)$ such that the operator

$$
A: L^{p-\varepsilon}\left(v(x)^{1+\delta} x^{\gamma}\right) \rightarrow L^{q-\varepsilon q / p}\left(w(x)^{1+\delta} x^{\delta(1-q / p)} x^{\gamma q / p}\right)
$$

is also bounded for all $\varepsilon, \delta, \gamma \in\left[0, \varepsilon_{0}\right)$. 


\section{Theorem 1.2}

Let $1<p \leq q<+\infty$ and let $v, w$ be weights on $(0,+\infty)$ such that (2) and (3) hold. Assume that the averaging operator

$$
A: L^{p-\varepsilon}\left(v(x)^{1+\delta} x^{\gamma}\right) \rightarrow L^{q-\varepsilon q / p}\left(w(x)^{1+\delta} x^{\delta(1-q / p)} x^{\gamma q / p}\right)
$$

is bounded for some $\varepsilon \in[0, p-1), \delta \geq 0$ and $\gamma \geq 0$. Then the operator $A: L^{p}(v) \rightarrow$ $L^{q}(w)$ is also bounded.

Remark 1.3 Assumptions of Theorem 1.1 (or Theorem 1.2) ensure that

$$
\left(\int_{r}^{+\infty} w(t) t^{-q} \mathrm{~d} t\right)^{1 / q}\left(\int_{0}^{r} v(t)^{1-p^{\prime}} \mathrm{d} t\right)^{1 / p^{\prime}} \approx 1 \text { for all } r>0,
$$

which means that $(w, v)$ is the optimal couple of weights for which (1) holds.

Note also that assumption (3) is satisfied when $w=v$ and $q=p$.

Theorem 1.1 is a particular case of the following assertion.

\section{Theorem 1.4}

Let $1<p \leq q<+\infty$ and let $v, w$ be weights on $(0,+\infty)$ such that (2) and (3) hold. Assume that the averaging operator $A: L^{p}(v) \rightarrow L^{q}(w)$ is bounded. Then there exist $p_{0} \in(1, p)$ and $\varepsilon_{0}>0$ such that the operator

$$
A: L^{P}\left(v(x)^{1+\delta} x^{\gamma}\right) \rightarrow L^{Q}\left(w(x)^{1+\delta} x^{\delta(1-Q / P)} x^{\gamma Q / P}\right)
$$

is also bounded for all $P \in\left(p_{0},+\infty\right)$ and for every $\delta, \gamma \in\left[0, \varepsilon_{0}\right)$, where $Q=P q / p$.

Remark 1.5 If $1<p<+\infty$ and $v$ is a weight on $(0,+\infty)$, then we write $v \in M_{p}$ when the averaging operator $A$ is bounded on the space $L^{p}(v)$, that is, when (1) holds with $q=p$ and $w=v$. Let $A_{p}, 1<p<+\infty$, be the $A_{p}$-class of B. Muckenhoupt of those weights $v$ on $(0,+\infty)$ for which the Hardy-Littlewood maximal operator associated with the interval $(0,+\infty)$ is bounded on the space $L^{p}(v)$. Recall that $A_{p} \subset M_{p}$. Denote by $C_{p}, 1<p<+\infty$, the $C_{p}$-class of Calderón (introduced in [1]) of those weights $v$ on $(0,+\infty)$ for which both the operator $A$ and its adjoint operator $A^{\prime}$ are bounded on the space $L^{p}(v)$.

If (2) holds with $\rho=0$, then $v$ is equivalent to a non-decreasing function on $(0,+\infty)$. It is known ( $c f$. [4, Theorem 6.1] or [3, Proposition 2.3]) that a non-decreasing weight $v$ satisfies $v \in M_{p}$ if and only if it belongs to the $A_{p}$-class. Moreover, it can be shown that a non-decreasing weight $v$ from the class $M_{p}$ also belongs to the $C_{p}$-class. Since

$$
\begin{array}{ll}
v \in A_{p} \Longrightarrow v \in A_{p-\varepsilon} & \text { for some } \varepsilon \in(0, p-1), \\
v \in A_{p} \Longrightarrow v^{1+\varepsilon} \in A_{p} & \text { for some } \varepsilon>0, \\
v \in A_{p} \Longrightarrow v \in A_{q} & \text { for all } q \in[p,+\infty], \\
v \in C_{p} \Longrightarrow v(x) x^{\varepsilon} \in M_{p} & \text { for some } \varepsilon>0
\end{array}
$$

(cf. [6, 5] for the first three implications, and [1, Proposition 2.4] for the last one), Theorem 1.4 with $\rho=0$ also follows from properties of weights $v \in A_{p} \cap C_{p}$. (This is 
clear if, in addition, $p=q$ in Theorem 1.4. If $p<q$, one can show that it is again true due to condition (3).)

On the other hand, there are weights in the $M_{p}$-class which satisfy (2) but which do not belong to $A_{p} \cap C_{p}$. A simple example is $v(t)=t^{\beta}, t>0$, with $\beta \leq-1$. (Note that the weight $v(t)=t^{\beta}, t>0$, with $\beta \in \mathbb{R}$, belongs to the $A_{p}$-class or the $C_{p}$-class if and only if $-1<\beta<p-1$. However, $v$ belongs to the $M_{p^{-}}$class if and only if $\beta<p-1$.) Another example is $v(t)=t^{\beta}(1+|\ln t|)^{\alpha}, t>0$, with $\beta \leq-1$ and $\alpha \in \mathbb{R}$.

Remark 1.6 Denote by $D_{p}, 1<p<+\infty$, the subset of the $M_{p^{-}}$class consisting of those weights $v$ on $(0,+\infty)$ which satisfy condition (2). In particular, our results imply that the $D_{p}$-class possesses properties similar to those of the $A_{p}$-class. Namely,

$$
\begin{array}{lll}
v \in D_{p} \Longrightarrow v \in D_{p-\varepsilon} & & \text { for some } \varepsilon \in(0, p-1), \\
v \in D_{p} \Longrightarrow v^{1+\varepsilon} \in D_{p} & & \text { for some } \varepsilon>0, \\
v \in D_{p} \Longrightarrow v \in D_{q} & & \text { for all } q \in[p,+\infty) .
\end{array}
$$

Moreover,

$$
v \in D_{p} \Longrightarrow v(x) x^{\varepsilon} \in D_{p} \quad \text { for some } \varepsilon>0 .
$$

It is well-known that a weight $v \in A_{p}$ possesses a better integrability than that mentioned in the $A_{p}$-condition and that such a weight $v$ satisfies a reverse Hölder inequality. Implication (4) shows that also a weight $v \in D_{p}$ possesses better integrability properties than those mentioned in the definition of the $D_{p}$-class $(c f$. (1) with $w=v$ and $q=p$ ). It is even possible to prove that certain reverse Hölder inequalities hold for such a weight (cf. [8]).

The paper is organized as follows. In Section 2 we prove Theorem 1.1. Section 3 is devoted to the proof of Theorem 1.2. Finally, in Section 4 we prove Theorem 1.4.

Acknowledgement. We would like to thank Mario Milman for the information concerning properties of $C_{p}$-weights.

\section{Proof of Theorem 1.1}

To prove Theorem 1.1, we shall use the following two assertions.

Lemma A (see [7, Lemma 2])

Let $\varphi:(0,+\infty) \rightarrow(0,+\infty)$. If there is a constant $c_{0}>0$ such that

$$
\int_{r}^{+\infty} \varphi(t) \frac{\mathrm{d} t}{t} \leq c_{0} \varphi(r) \quad \text { for all } r>0,
$$

then there exist positive constants $\alpha_{1}$ and $c$ such that

$$
\int_{r}^{+\infty} \varphi(t) t^{\alpha} \frac{\mathrm{d} t}{t} \leq c \varphi(r) r^{\alpha} \quad \text { for all } r>0 \text { and } \alpha \in\left[0, \alpha_{1}\right) .
$$


Remark 2.1 In fact, it is proved in [7] that the last inequality holds for all $r>0$ and some $\alpha>0$. However, checking the [7, proof of Lemma 2], one can see that Lemma A holds, e.g., with $\alpha_{1}=\left(2 c_{0}\right)^{-1}$ (and then one can put $c=2 c_{0}$ ), where $c_{0}$ is the constant in $(5)$.

\section{Lemma $A^{*}$}

Let $\varphi:(0,+\infty) \rightarrow(0,+\infty)$. If there is a constant $c_{0}>0$ such that

$$
\int_{0}^{r} \varphi(t) \frac{\mathrm{d} t}{t} \leq c_{0} \varphi(r) \quad \text { for all } r>0,
$$

then there exist positive constants $\beta_{1}$ and $c$ such that

$$
\int_{0}^{r} \varphi(t) t^{-\beta} \frac{\mathrm{d} t}{t} \leq c \varphi(r) r^{-\beta} \text { for all } r>0 \text { and } \beta \in\left[0, \beta_{1}\right) \text {. }
$$

Proof. Lemma $\mathrm{A}^{*}$ can be obtained from Lemma A by the change of variables $t \mapsto$ $t^{-1}$.

In addition, we shall also need the following lemma.

\section{Lemma B}

Let $1<p \leq q<+\infty$ and let $v, w$ be weights on $(0,+\infty)$ such that (2) and (3) hold. Assume that the averaging operator $A: L^{p}(v) \rightarrow L^{q}(w)$ is bounded. Then there exists a positive constant $\alpha_{0}$ such that

$$
\int_{0}^{r}\left[v(t) t^{\alpha}\right]^{1-p^{\prime}} \mathrm{d} t \approx\left[v(r) r^{\alpha+1-p}\right]^{1-p^{\prime}}
$$

and

$$
\int_{r}^{+\infty} w(t) t^{\alpha-q} \mathrm{~d} t \approx w(r) r^{\alpha+1-q}
$$

for all $r>0$ and $\alpha \in\left[0, \alpha_{0}\right)$.

Proof. Assume that all the assumptions of Lemma B are satisfied. Since the function $t \mapsto v(t) t^{\alpha+\rho}, \alpha \geq 0$, is equivalent to a non-decreasing function on $(0,+\infty)$,

$$
\begin{aligned}
\int_{0}^{r}\left[v(t) t^{\alpha}\right]^{1-p^{\prime}} \mathrm{d} t & =\int_{0}^{r}\left[v(t) t^{\alpha+\rho}\right]^{1-p^{\prime}} t^{\rho\left(p^{\prime}-1\right)} \mathrm{d} t \\
& \gtrsim\left[v(r) r^{\alpha+\rho}\right]^{1-p^{\prime}} \int_{0}^{r} t^{\rho\left(p^{\prime}-1\right)} \mathrm{d} t \\
& \approx\left[v(r) r^{\alpha+\rho}\right]^{1-p^{\prime}} r^{\rho\left(p^{\prime}-1\right)+1} \\
& =\left[v(r) r^{\alpha+1-p}\right]^{1-p^{\prime}} \text { for all } r>0 \text { and } \alpha \geq 0 .
\end{aligned}
$$

Consequently, we obtain from (1), (8) (with $\alpha=0$ ) and (3) that

$$
\int_{r}^{+\infty} w(t) t^{-q} \mathrm{~d} t \leq \frac{B^{q}}{\left(\int_{0}^{r} v(t)^{1-p^{\prime}} \mathrm{d} t\right)^{q / p^{\prime}}} \precsim v(r)^{q / p} r^{-q / p^{\prime}} \approx w(r) r^{1-q}
$$


for all $r>0$. Setting $\varphi(r)=w(r) r^{1-q}$, we can rewrite estimate (9) in the form

$$
\int_{r}^{+\infty} \varphi(t) \frac{\mathrm{d} t}{t} \lesssim \varphi(r) \quad \text { for all } r>0
$$

Thus, by Lemma A, there exist constants $\alpha_{1}>0$ and $c>0$ such that

$$
\int_{r}^{+\infty} w(t) t^{\alpha-q} \mathrm{~d} t=\int_{r}^{+\infty} \varphi(t) t^{\alpha} \frac{\mathrm{d} t}{t} \leq c \varphi(r) r^{\alpha}=c w(r) r^{\alpha+1-q}
$$

for all $r>0$ and $\alpha \in\left[0, \alpha_{1}\right)$.

On the other hand, using (3) and the fact that the function $t \mapsto\left[v(t) t^{\rho+1}\right]^{q / p} t^{\alpha}$, $\alpha \geq 0$, is equivalent to a non-decreasing function on $(0,+\infty)$, we arrive at

$$
\begin{aligned}
\int_{r}^{+\infty} w(t) t^{\alpha-q} \mathrm{~d} t & \approx \int_{r}^{+\infty}\left[v(t) t^{\rho+1}\right]^{q / p} t^{\alpha} t^{-\rho q / p-q-1} \mathrm{~d} t \\
& \succsim\left[v(r) r^{\rho+1}\right]^{q / p} r^{\alpha} \int_{r}^{+\infty} t^{-\rho q / p-q-1} \mathrm{~d} t \\
& \approx[v(r) r]^{q / p} r^{\alpha} r^{-q} \\
& =w(r) r^{\alpha+1-q} \text { for all } r>0 \text { and } \alpha \geq 0 .
\end{aligned}
$$

Thus, (10) and (11) imply that (7) holds for all $r>0$ and $\alpha \in\left[0, \alpha_{1}\right)$.

Condition (1) and the first three estimates in (11) (with $\alpha=0$ ) yield

$$
\begin{aligned}
\int_{0}^{r} v(t)^{1-p^{\prime}} \mathrm{d} t & \leq \frac{B^{p^{\prime}}}{\left(\int_{r}^{+\infty} w(t) t^{-q} \mathrm{~d} t\right)^{p^{\prime} / q}} \\
& \precsim \frac{1}{\left([v(r) r]^{q / p} r^{-q}\right)^{p^{\prime} / q}} \\
& =v(r)^{1-p^{\prime}} r \quad \text { for all } r>0 .
\end{aligned}
$$

Rewriting (12) in terms of the function $\psi(t)=v(t)^{1-p^{\prime}} t, t>0$, and applying Lemma $\mathrm{A}^{*}$, we obtain that there are constants $\beta_{1}>0$ and $c_{1}>0$ such that

$$
\int_{0}^{r} v(t)^{1-p^{\prime}} t^{-\beta} \mathrm{d} t \leq c_{1} v(r)^{1-p^{\prime}} r^{1-\beta}
$$

for all $r>0$ and $\beta \in\left[0, \beta_{1}\right)$. Setting $\alpha=\beta /\left(p^{\prime}-1\right)$ and $\alpha_{2}=\beta_{1} /\left(p^{\prime}-1\right)$, we can rewrite (13) in the form

$$
\int_{0}^{r}\left[v(t) t^{\alpha}\right]^{1-p^{\prime}} \mathrm{d} t \precsim\left[v(r) r^{\alpha+1-p}\right]^{1-p^{\prime}}
$$

for all $r>0$ and $\alpha \in\left[0, \alpha_{2}\right)$. Together with (8), this shows that (6) holds for all $r>0$ and $\alpha \in\left[0, \alpha_{2}\right)$.

Now, it suffices to put $\alpha_{0}=\min \left\{\alpha_{1}, \alpha_{2}\right\}$. 
Remark 2.2 On using (3), one can rewrite (7) as

$$
\int_{r}^{+\infty} w(t) t^{\alpha-q} \mathrm{~d} t \approx v(r)^{q / p} r^{\alpha-q+q / p}
$$

for all $r>0$ and $\alpha \in\left[0, \alpha_{0}\right)$.

Remark 2.3 Let all the assumptions of Lemma B be satisfied. Then the operator

$$
A: L^{p}\left(v(x) x^{\alpha}\right) \rightarrow L^{q}\left(w(x) x^{\alpha q / p}\right)
$$

is also bounded for all $\alpha \in\left[0, \alpha_{0} p / q\right)$. Indeed, making use of estimates (6) and (14) (with $\alpha$ replaced by $\alpha q / p$ ), we see that (1) holds with $v(t)$ replaced by $v(t) t^{\alpha}$ and with $w(t)$ replaced by $w(t) t^{\alpha q / p}$ for all $\alpha \in\left[0, \alpha_{0} p / q\right)$.

Proof of Theorem 1.1. Let the assumptions of Theorem 1.1 be satisfied. By (6) and (7) (with $\alpha=0$ ), for all $r>0$,

$$
\int_{0}^{r} v(t)^{1-p^{\prime}} \mathrm{d} t \approx v(r)^{1-p^{\prime}} r
$$

and

$$
\int_{r}^{+\infty} w(t) t^{-q} \mathrm{~d} t \approx w(r) r^{1-q}
$$

Take $\delta, \gamma \geq 0, \varepsilon \in[0, p-1)$ and put $p(\varepsilon):=p-\varepsilon, q(\varepsilon):=q-\varepsilon q / p$. Clearly, $p(\varepsilon), p(\varepsilon)^{\prime} \in(1,+\infty), p^{\prime}-p(\varepsilon)^{\prime} \leq 0$ and $p(\varepsilon) / p=q(\varepsilon) / q=1-\varepsilon / p$. Thus,

$$
\kappa:=\frac{p^{\prime}-p(\varepsilon)^{\prime}}{1-p^{\prime}}+\delta \frac{1-p(\varepsilon)^{\prime}}{1-p^{\prime}} \geq 0
$$

and the function

$$
t \mapsto\left(\int_{0}^{t} v(\tau)^{1-p^{\prime}} \mathrm{d} \tau\right)^{\kappa}
$$

is non-decreasing on $(0,+\infty)$. Consequently, applying (15), we obtain

$$
\begin{aligned}
\int_{0}^{r}\left[v(t)^{1+\delta} t^{\gamma}\right]^{1-p(\varepsilon)^{\prime}} \mathrm{d} t & =\int_{0}^{r} v(t)^{1-p^{\prime}} v(t)^{\kappa\left(1-p^{\prime}\right)} t^{\gamma\left(1-p(\varepsilon)^{\prime}\right)} \mathrm{d} t \\
& \approx \int_{0}^{r} v(t)^{1-p^{\prime}}\left(t^{-1} \int_{0}^{t} v(\tau)^{1-p^{\prime}} \mathrm{d} \tau\right)^{\kappa} t^{\gamma\left(1-p(\varepsilon)^{\prime}\right)} \mathrm{d} t \\
& \leq\left(\int_{0}^{r} v(\tau)^{1-p^{\prime}} \mathrm{d} \tau\right)^{\kappa} \int_{0}^{r} v(t)^{1-p^{\prime}} t^{-\kappa+\gamma\left(1-p(\varepsilon)^{\prime}\right)} \mathrm{d} t \\
& \approx v(r)^{\kappa\left(1-p^{\prime}\right)} r^{\kappa} \int_{0}^{r}\left[v(t) t^{\alpha}\right]^{1-p^{\prime}} \mathrm{d} t
\end{aligned}
$$

where

$$
\begin{aligned}
\alpha & \equiv \alpha(\varepsilon, \delta, \gamma) \\
& :=\frac{-\kappa+\gamma\left(1-p(\varepsilon)^{\prime}\right)}{1-p^{\prime}} \\
& =\frac{p^{\prime}-p(\varepsilon)^{\prime}}{\left(1-p^{\prime}\right)\left(p^{\prime}-1\right)}+\delta \frac{1-p(\varepsilon)^{\prime}}{\left(1-p^{\prime}\right)\left(p^{\prime}-1\right)}+\gamma \frac{1-p(\varepsilon)^{\prime}}{1-p^{\prime}} \geq 0
\end{aligned}
$$


Since the function $(\varepsilon, \delta, \gamma) \mapsto \alpha(\varepsilon, \delta, \gamma)$ is non-negative and continuous on the set $[0, p-1) \times[0,+\infty) \times[0,+\infty)$ and $\alpha(0,0,0)=0$, there is $\varepsilon_{1} \in(0, p-1)$ such that $\alpha(\varepsilon, \delta, \gamma) \in\left[0, \alpha_{0}\right)$ provided that $\varepsilon, \delta, \gamma \in\left[0, \varepsilon_{1}\right)$, where the number $\alpha_{0}$ is from Lemma B. Therefore, (17) and (6) imply that

$$
\int_{0}^{r}\left[v(t)^{1+\delta} t^{\gamma}\right]^{1-p(\varepsilon)^{\prime}} \mathrm{d} t \lesssim v(r)^{(1+\delta)\left(1-p(\varepsilon)^{\prime}\right)} r^{\gamma\left(1-p(\varepsilon)^{\prime}\right)+1}
$$

for all $r>0$ and $\varepsilon, \delta, \gamma \in\left[0, \varepsilon_{1}\right)$. Hence,

$$
\left(\int_{0}^{r}\left[v(t)^{1+\delta} t^{\gamma}\right]^{1-p(\varepsilon)^{\prime}} \mathrm{d} t\right)^{1 / p(\varepsilon)^{\prime}} \lesssim v(r)^{-(1+\delta) / p(\varepsilon)} r^{-\gamma / p(\varepsilon)} r^{1 / p(\varepsilon)^{\prime}}
$$

for all $r>0$ and $\varepsilon, \delta, \gamma \in\left[0, \varepsilon_{1}\right)$.

Applying (7) (with $\alpha=0$ ), the fact that the function

$$
t \mapsto\left(\int_{t}^{+\infty} w(\tau) \tau^{-q} \mathrm{~d} \tau\right)^{\delta} t^{\delta(1-q / p)}, \quad \delta \leq 0,
$$

is non-increasing on $(0,+\infty)$ and (14) (with $\alpha=0)$, we get

$$
\begin{aligned}
& \int_{r}^{+\infty} w(t)^{1+\delta} t^{\delta(1-q / p)} t^{\gamma q / p} t^{-q(\varepsilon)} \mathrm{d} t \\
& \approx \int_{r}^{+\infty} w(t)\left(t^{q-1} \int_{t}^{+\infty} w(\tau) \tau^{-q} \mathrm{~d} \tau\right)^{\delta} t^{(\gamma+\varepsilon) q / p-q} t^{\delta(1-q / p)} \mathrm{d} t \\
& \leq\left(\int_{r}^{+\infty} w(\tau) \tau^{-q} \mathrm{~d} \tau\right)^{\delta} r^{\delta(1-q / p)} \int_{r}^{+\infty} w(t) t^{(\gamma+\varepsilon) q / p+\delta(q-1)-q} \mathrm{~d} t \\
& \approx\left[v(r)^{q / p} r^{-q+q / p}\right]^{\delta} r^{\delta(1-q / p)} \int_{r}^{+\infty} w(t) t^{(\gamma+\varepsilon) q / p+\delta(q-1)-q} \mathrm{~d} t .
\end{aligned}
$$

Now, using (14) (with $(\gamma+\varepsilon) q / p+\delta(q-1)$ instead of $\alpha$ ) to estimate the last integral, we arrive at

$$
\int_{r}^{+\infty} w(t) t^{(\gamma+\varepsilon) q / p+\delta(q-1)-q} \mathrm{~d} t \approx v(r)^{q / p} r^{(\gamma+\varepsilon+1) q / p+\delta(q-1)-q}
$$

for all $r>0$ provided that $(\gamma+\varepsilon) q / p+\delta(q-1) \in\left[0, \alpha_{0}\right)$. Therefore, (19) and (20) imply that

$$
\left(\int_{r}^{+\infty} w(t)^{1+\delta} t^{\delta(1-q / p)} t^{\gamma q / p} t^{-q(\varepsilon)} \mathrm{d} t\right)^{1 / q(\varepsilon)} \precsim v(r)^{(1+\delta) / p(\varepsilon)} r^{\gamma / p(\varepsilon)} r^{-1 / p(\varepsilon)^{\prime}}
$$

for all $r>0$ and $\varepsilon, \delta, \gamma \in\left[0, \varepsilon_{2}\right)$, where $\varepsilon_{2}:=\min \left\{\alpha_{0} p /(3 q), \alpha_{0} /(3(q-1))\right\}$.

Putting $\varepsilon_{0}=\min \left\{\varepsilon_{1}, \varepsilon_{2}\right\}$ and using estimates (18) and (21) in (1) (with $w(t)$, $v(t), q$ and $p$ replaced by $w(t)^{1+\delta} t^{\delta(1-q / p)} t^{\gamma q / p}, v(t)^{1+\delta} t^{\gamma}, q(\varepsilon)$ and $p(\varepsilon)$, respectively), we obtain the desired result. 


\section{Proof of Theorem 1.2}

Assume that the assumptions of Theorem 1.2 are satisfied. Put $p(\varepsilon):=p-\varepsilon$ and $q(\varepsilon):=q-\varepsilon q / p$. The Hölder inequality with the exponents

$$
\frac{\left(p(\varepsilon)^{\prime}-1\right)(1+\delta)}{\left(p^{\prime}-1\right)} \text { and } \frac{\left(p(\varepsilon)^{\prime}-1\right)(1+\delta)}{\left(p(\varepsilon)^{\prime}-1\right)(1+\delta)-\left(p^{\prime}-1\right)}
$$

implies that, for all $r>0$,

$$
\int_{0}^{r} v(t)^{1-p^{\prime}} \mathrm{d} t \leq\left(\int_{0}^{r}\left[v(t)^{1+\delta}\right]^{1-p(\varepsilon)^{\prime}} \mathrm{d} t\right)^{\frac{p^{\prime}-1}{\left(p(\varepsilon)^{\prime}-1\right)(1+\delta)}} r^{\frac{\left(p(\varepsilon)^{\prime}-1\right)(1+\delta)-p^{\prime}+1}{\left(p(\varepsilon)^{\prime}-1\right)(1+\delta)}} .
$$

Using the fact that the function $t \mapsto t^{\gamma\left(p(\varepsilon)^{\prime}-1\right)}$ is non-decreasing on the interval $(0,+\infty)$, we obtain

$$
\int_{0}^{r}\left[v(t)^{1+\delta}\right]^{1-p(\varepsilon)^{\prime}} \mathrm{d} t \leq r^{\gamma\left(p(\varepsilon)^{\prime}-1\right)} \int_{0}^{r}\left[v(t)^{1+\delta} t^{\gamma}\right]^{1-p(\varepsilon)^{\prime}} \mathrm{d} t \quad \text { for all } r>0 .
$$

Fix $\bar{\rho} \geq \max \{\rho(1+\delta)-\gamma, 0\}$. One can easily verify that (2) and (3) holds with $v(x) x^{\rho}$, $w(x), v(x), q$ and $p$ replaced by $\left(v(x)^{1+\delta} x^{\gamma}\right) x^{\bar{\rho}}, w(x)^{1+\delta} x^{\delta(1-q / p)} x^{\gamma q / p}, v(x)^{1+\delta} x^{\gamma}, q(\varepsilon)$ and $p(\varepsilon)$, respectively. Thus, we can apply Lemma B (with $v(x) x^{\rho}, w(x), v(x), q$ and $p$ replaced by $\left(v(x)^{1+\delta} x^{\gamma}\right) x^{\bar{\rho}}, w(x)^{1+\delta} x^{\delta(1-q / p)} x^{\gamma q / p}, v(x)^{1+\delta} x^{\gamma}, q(\varepsilon)$ and $p(\varepsilon)$, respectively). Hence, taking $\alpha=0$ in (6) and (7), we obtain, for all $r>0$,

$$
\int_{0}^{r}\left[v(t)^{1+\delta} t^{\gamma}\right]^{1-p(\varepsilon)^{\prime}} \mathrm{d} t \approx\left[v(r)^{1+\delta} r^{\gamma}\right]^{1-p(\varepsilon)^{\prime}} r
$$

and

$$
\int_{r}^{+\infty} w(t)^{1+\delta} t^{\delta(1-q / p)} t^{\gamma q / p} t^{-q(\varepsilon)} \mathrm{d} t \approx w(r)^{1+\delta} r^{\delta(1-q / p)} r^{\gamma q / p} r^{1-q(\varepsilon)}
$$

Combining estimates (22)-(24), we arrive at

$$
\left(\int_{0}^{r} v(t)^{1-p^{\prime}} \mathrm{d} t\right)^{1 / p^{\prime}} \lesssim v(r)^{-1 / p} r^{1 / p^{\prime}} \quad \text { for all } r>0
$$

On the other hand, Hölder's inequality with the exponents $1+\delta$ and $(1+\delta) / \delta$ gives

$$
\int_{r}^{+\infty} w(t) t^{-q} \mathrm{~d} t \leq\left(\int_{r}^{+\infty} w(t)^{1+\delta} t^{\delta(1-q / p)} t^{\gamma q / p} t^{-q(\varepsilon)} \mathrm{d} t\right)^{1 /(1+\delta)}\left(r^{\frac{q}{p}-\frac{\gamma q}{\delta p}-\frac{\varepsilon q}{\delta p}-q}\right)^{\delta /(1+\delta)}
$$

which, together with (25) and (3), implies that

$$
\left(\int_{r}^{+\infty} w(t) t^{-q} \mathrm{~d} t\right)^{1 / q} \lesssim w(r)^{1 / q} r^{-1 / q^{\prime}} \approx v(r)^{1 / p} r^{-1 / p^{\prime}} \quad \text { for all } r>0 .
$$

Estimates (26) and (27) used in (1) yield the desired result. 


\section{Proof of Theorem 1.4}

With respect to Theorem 1.1, it is sufficient to prove that the operator $A: L^{P}(v(x)) \rightarrow L^{Q}(w(x))$ is bounded if $p<P<+\infty$ and $Q / P=q / p$.

Using the monotonicity of the function $t \mapsto t^{q-Q}, t>0$, and (14) (with $\alpha=0$ ), we obtain

$$
\begin{aligned}
\left(\int_{r}^{+\infty} w(t) t^{-Q} \mathrm{~d} t\right)^{1 / Q} & \leq\left(r^{q-Q} \int_{r}^{+\infty} w(t) t^{-q} \mathrm{~d} t\right)^{1 / Q} \\
& \approx\left(r^{q-Q} v(r)^{q / p} r^{-q+q / p}\right)^{1 / Q} \\
& =v(r)^{1 / P} r^{-1 / P^{\prime}} \text { for all } r>0 .
\end{aligned}
$$

Moreover, the Hölder inequality (with the exponents $\frac{1-p^{\prime}}{1-P^{\prime}}$ and $\frac{1-p^{\prime}}{P^{\prime}-p^{\prime}}$ ) and (6) (with $\alpha=0$ ) imply that

$$
\begin{aligned}
\left(\int_{0}^{r} v(t)^{1-P^{\prime}} \mathrm{d} t\right)^{1 / P^{\prime}} & \leq\left(\int_{0}^{r} v(t)^{1-p^{\prime}} \mathrm{d} t\right)^{\left(1-P^{\prime}\right) /\left(\left(1-p^{\prime}\right) P^{\prime}\right)} r^{\left(P^{\prime}-p^{\prime}\right) /\left(\left(1-p^{\prime}\right) P^{\prime}\right)} \\
& \approx\left[v(r)^{1-p^{\prime}} r\right]^{\left(1-P^{\prime}\right) /\left(\left(1-p^{\prime}\right) P^{\prime}\right)} r^{\left(P^{\prime}-p^{\prime}\right) /\left(\left(1-p^{\prime}\right) P^{\prime}\right)} \\
& =v(r)^{-1 / P} r^{1 / P^{\prime}} \text { for all } r>0 .
\end{aligned}
$$

Consequently, the result follows from (1) (with $p$ and $q$ replaced by $P$ and $Q$, respectively).

\section{References}

1. J. Bastero, M. Milman, and F.J. Ruiz, On the Connection Between Weighted Norm Inequalities, Commutators and Real Interpolation, Mem. Amer. Math. Soc. 731, Providence, 2001.

2. J.S. Bradley, Hardy inequalities with mixed norms, Canad. Math. Bull. 21 (1978), 405-408.

3. J. Cerdà and J. Martín, Weighted Hardy inequalities and Hardy transforms of weights, Studia Math. 139 (2000), 189-196.

4. D. Cruz-Uribe, SFO, Piecewise monotonic doubling measures, Rocky Mountain J. Math. 26 (1996), 545-583.

5. J. García-Cuerva and J.L. Rubio de Francia, Weighted Norm Inequalities and Related Topics, North-Holland Mathematics Studies 116, North-Holland, Amsterdam, 1985.

6. B. Muckenhoupt, Weighted norm inequalities for the Hardy maximal function, Trans. Amer. Math. Soc. 165 (1972), 207-226.

7. E. Nakai, Hardy-Littlewood maximal operator, singular integral operators and the Riesz potentials on generalized Morrey spaces, Math. Nachr. 166 (1994), 95-103.

8. B. Opic, The averaging integral operator between weighted Lebesgue spaces and reverse Hölder inequalities, (preprint 187, IM AS CR, Prague, 2009). To appear in Complex. Var. Elliptic Equ.

9. B. Opic and A. Kufner, Hardy-Type Inequalities, Pitman Research Notes in Mathematics Series 219, Longman Scientific \& Technical, Harlow, 1990. 